\title{
Localized solutions for the finite difference semi-discretization of the wave equation
}

\section{Solutions localisées pour la semi-discrétisation par différences finies de l'équation des ondes}

\author{
Aurora Marica $^{\mathrm{b}}$, Enrique Zuazua ${ }^{\mathrm{a}, \mathrm{b}}$ \\ a Ikerbasque, Basque Foundation for Sciences, Basque Country, Spain \\ ${ }^{\mathrm{b}}$ BCAM - Basque Center for Applied Mathematics, Bizkaia Technology Park, 500, E-48160, Derio, Basque Country, \\ Spain
}

\begin{abstract}
We study the propagation properties of the solutions of the finite-difference space semi-discrete wave equation on an uniform grid of the whole Euclidean space. We provide a construction of high frequency wave packets that propagate along the corresponding bi-characteristic rays of Geometric Optics with a group velocity arbitrarily close to zero. Our analysis is motivated by control theoretical issues. In particular, the continuous wave equation has the so-called observability property: for a sufficiently large time, the total energy of its solutions can be estimated in terms of the energy concentrated in the exterior of a compact set. This fails to be true, uniformly on the mesh-size parameter, for the semi-discrete schemes and the observability constant blows-up at an arbitrarily large polynomial order. Our contribution consists in providing a rigorous derivation of those wave packets and in analyzing their behavior near that ray, by taking into account the subtle added dispersive effects that the numerical scheme introduces.
\end{abstract}

\section{Résumé}

On étudie les propriétés de propagation des solutions de l'équation des ondes semi-discretisée en espace par différences finies sur une grille uniforme dans tout l'espace euclidien. On réalise une construction de paquets d'ondes concentrés à haute fréquence qui se propagent le long des rayons bicaractéristiques de l'Optique Géométrique à une vitesse de groupe arbitrairement petite. Notre analyse est motivée par la théorie du contrôle. Plus précisement, l'équation des ondes continue vérifie la propriété d'observabilité : pour un temps suffisament grand, l'énergie totale de ses solutions peut être estimée en fonction de l'énergie localisée à l'extérieur d'un ensemble compact. Cette propriété n'est pas verifiée de manière uniforme par rapport au pas de discrétisation pour le schéma semidiscret pour un temps fini quelconque, si bien que la constante d'observabilité semi-discrète diverge avec un taux polynomial arbitraire. Nous donnons une construction précise de ces paquets d'ondes et decrivons l'effet dispersif rajouté que le schéma numérique génère autour du rayon de propagation. 


\section{Version française abrégée}

Il est bien connu (cf. [11]) que les solutions du problème de Cauchy pour l'équation des ondes continue $d$-dimensionnelle vérifient la propriété d'observabilité : pour un temps suffisament grand, l'énergie totale des solutions peut être estimée en fonction de l'énergie localisée à l'extérieur d'un ensemble compact. Par la méthode d'unicité de Hilbert (HUM) introduit dans [5], cette propriété est equivalente à un résultat de contrôlabilité exacte par un contrôle localisé dans le complémentaire du même ensemble compact.

Dans cet article, on considère le problème de Cauchy associé à l'équation des ondes $d$-dimensionnelle semi-discretisée en espace par un schéma centré en différences finies dans un maillage uniforme. Nous avons deux objectifs. Le premier est de montrer l'éxistence de paquets d'ondes concentrés autour d'un nombre d'onde fixé a priori, à haute fréquence, propageant avec une vitesse de groupe arbitrairement petite autour des rayons bicharactéristiques du schéma semi-discret et donc peu visibles depuis le domaine d'observation. On déduit dans [2] que la constante d'observabilité semi-discrète diverge au moins d'une manière polynomiale arbitraire, et ce pour tout temps d'observabilité fini. Le deuxième objectif est de donner une forme asymptotique précise pour ces paquets d'ondes, de façon à mettre en évidence la dispersion numérique qui n'apparait pas dans le modèle continu.

Notre résultat complète la littérature existante sur l'observabilité et le contrôle des schémas d'approximation de l'équation des ondes. En particulier, dans [10], pour la semi-discrétisation de l'équation des ondes par différences finies dans des domaines bornés comme les cubes $d$-dimensionnels, il a été prouvé que l'inégalité d'observabilité explose au sens où la constante d'observabilité explose lorsque le pas du maillage tend vers zéro. Dans [7], on montre aussi que pour l'équation des ondes unidimensionnelle sur un intervalle borné observée par le bord, la constante d'observabilité diverge de manière exponentielle. Ce type de phénomène pathologique apparaît aussi au niveau des estimations dispersives de Strichartz associées à l'équation de Schrödinger semi-discretisée en espace par différences finies, cf. [4].

L'avantage de notre construction de paquets d'ondes numériques à haute fréquence est qu'elle peut être généralisée à d'autres approximations plus sophistiquées de l'équation des ondes comme celles obtenues par les méthodes de Galerkin discontinus ou par des éléments finis classiques d'ordre supérieur, où le symbole "numérique" en Fourier du Laplacien est une matrice donnant lieu à plusieurs relations de dispersion, cf. [6].

En outre, les paquets d'ondes que nous construisons sont aussi localisés dans l'espace physique et peuvent donc être adaptés pour des problèmes aux limites dans des domaines bornés avec des conditions aux limites diverses.

\section{Introduction and problem formulation}

For a finite time $T>0$, consider the Cauchy problem associated to the $d$-dimensional wave equation:

$$
\begin{cases}\partial_{t}^{2} \phi-\Delta \phi=0, & x \in \mathbb{R}^{d}, t \in(0, T] \\ \phi(x, 0)=\phi^{0}(x), \partial_{t} \phi(x, 0)=\phi^{1}(x), & x \in \mathbb{R}^{d} .\end{cases}
$$

The problem (1) is well posed in $\dot{H}^{1}\left(\mathbb{R}^{d}\right) \times L^{2}\left(\mathbb{R}^{d}\right)\left(\dot{H}^{1}\left(\mathbb{R}^{d}\right)\right.$ is the completion of $C_{c}^{\infty}\left(\mathbb{R}^{d}\right)$ with respect to the semi-norm $\left.\|\cdot\|_{\dot{H}^{1}\left(\mathbb{R}^{d}\right)}^{2}=\|\nabla \cdot\|_{L^{2}\left(\mathbb{R}^{d}\right)}^{2}\right)$. The total energy of its solutions is conserved in time and is defined by $E\left(\phi^{0}, \phi^{1}\right)=\left(\|\phi(t)\|_{\dot{H}^{1}\left(\mathbb{R}^{d}\right)}^{2}+\left\|\partial_{t} \phi(t)\right\|_{L^{2}\left(\mathbb{R}^{d}\right)}^{2}\right) / 2$.

Email addresses: marica@bcamath.org (Aurora Marica), zuazua@bcamath.org (Enrique Zuazua). 
For all finite time $T>2$, there exists a constant $C(T)>0$ such that, for all $\left(\phi^{0}, \phi^{1}\right) \in \dot{H}^{1}\left(\mathbb{R}^{d}\right) \times L^{2}\left(\mathbb{R}^{d}\right)$, the following observability inequality holds (see [11]):

$$
E\left(\phi^{0}, \phi^{1}\right) \leqslant C(T) \int_{0}^{T} \int_{\Omega^{d}}\left|\partial_{t} \phi(x, t)\right|^{2} d x d t .
$$

The observation region $\Omega^{d}:=\mathbb{R}^{d} \backslash B^{d}(0,1)$ is the complement of the $d$-dimensional unit ball. This observability problem is motivated by controllability issues, since, by means of the Hilbert Uniqueness Method (HUM) introduced in [5], the inequality (2) is equivalent to the fact that, for all $T>2$ and all initial data $\left(u^{0}, u^{1}\right) \in \dot{H}^{1}\left(\mathbb{R}^{d}\right) \times L^{2}\left(\mathbb{R}^{d}\right)$, there exists a control function $f \in L^{2}\left(\Omega^{d} \times(0, T)\right)$ such that the solution of the inhomogeneous Cauchy problem

$$
\begin{cases}\partial_{t}^{2} u-\Delta u=f \chi_{\Omega^{d}}(x), & x \in \mathbb{R}^{d}, t \in(0, T] \\ u(x, 0)=u^{0}(x), \partial_{t} u(x, 0)=u^{1}(x), & x \in \mathbb{R}^{d}\end{cases}
$$

satisfies $u(x, T)=\partial_{t} u(x, T)=0$ for all $x \in \mathbb{R}^{d}$, where $\chi_{\Omega^{d}}$ is the characteristic function of the set $\Omega^{d}$.

These issues are by now well understood for the continuous wave equation. In particular, observability inequalities of the form (2) and several variants hold under the sharp Geometric Control Condition (GCC) (cf. [1]) requiring that all rays of Geometric Optics enter the observation subdomain during the observability time. As shown in [8], when the GCC is not satisfied, the observability property fails because of the existence of Gaussian beam solutions localized around a bi-characteristic ray that escape the observation region during the time interval $[0, T]$.

This paper is devoted to analyze these issues for the finite difference semi-discrete schemes. Given a mesh size $h>0$, we define an uniform grid of the whole Euclidean space by $x_{\mathbf{j}}=h \mathbf{j}, \mathbf{j} \in \mathbb{Z}^{d}$. We also introduce two discrete operators: the gradient $\nabla_{h}^{+}=\left(\partial_{h, k}^{+}\right)_{k=1, \cdots, d}$ and the Laplacian $\Delta_{h}$, with

$$
\partial_{h, k}^{+} \vec{f}=\frac{\vec{f}_{++\mathbf{e}_{k}}-\vec{f}}{h}, \quad \Delta_{h} \vec{f}=\frac{1}{h^{2}} \sum_{l=1}^{d}\left(\vec{f}_{+}+\mathbf{e}_{l}-2 \vec{f}+\vec{f} \cdot{ }_{-\mathbf{e}_{l}}\right),
$$

where $\vec{f}=\left(f_{\mathbf{j}}\right)_{\mathbf{j} \in \mathbb{Z}^{d}}$ is any sequence and $\left(\mathbf{e}_{l}\right)_{l=1}^{d}$ is the canonical basis in $\mathbb{R}^{d}$.

We consider the finite difference space semi-discretization of the wave equation (1):

$$
\begin{cases}\partial_{t}^{2} \phi_{\mathbf{j}}^{h}(t)-\Delta_{h} \phi_{\mathbf{j}}^{h}(t)=0, & \mathbf{j} \in \mathbb{Z}^{d}, t \in(0, T] \\ \phi_{\mathbf{j}}^{h}(0)=\phi_{\mathbf{j}}^{h, 0}, & \partial_{t} \phi_{\mathbf{j}}^{h}(0)=\phi_{\mathbf{j}}^{h, 1}, \mathbf{j} \in \mathbb{Z}^{d} .\end{cases}
$$

The problem (5) is well-posed in $\dot{\hbar}^{1} \times \ell^{2}$, with

$$
\ell^{2}=\left\{\vec{f} \text { s.t. }\|\vec{f}\|_{\ell^{2}}^{2}:=h^{d} \sum_{\mathbf{j} \in \mathbb{Z}^{d}}\left|f_{\mathbf{j}}\right|^{2}<\infty\right\} \text { and } \dot{\hbar}^{1}=\left\{\vec{f} \text { s.t. }\|\vec{f}\|_{\dot{\hbar}^{1}}^{2}:=\left\|\nabla_{h}^{+} \vec{f}\right\|_{\ell^{2}}^{2}=\sum_{k=1}^{d}\left\|\partial_{h, k}^{+} \vec{f}\right\|_{\ell^{2}}^{2}<\infty\right\} .
$$

The total energy associated to its solution is conserved in time, being defined by

$$
E_{h}\left(\vec{\phi}^{h, 0}, \vec{\phi}^{h, 1}\right)=\left(\left\|\vec{\phi}^{h}(t)\right\|_{\dot{\hbar}^{1}}^{2}+\left\|\partial_{t} \vec{\phi}^{h}(t)\right\|_{\ell^{2}}^{2}\right) / 2
$$

For a fixed $T>0$, consider the semi-discrete version of the observability inequality (2):

$$
E_{h}\left(\vec{\phi}^{h, 0}, \vec{\phi}^{h, 1}\right) \leq C_{h}(T) \int_{0}^{T}\left\|\partial_{t} \vec{\phi}(t)\right\|_{\ell^{2}\left(\Omega^{d}\right)}^{2} d t, \text { with }\left\|\partial_{t} \vec{\phi}(t)\right\|_{\ell^{2}\left(\Omega^{d}\right)}^{2}=h^{d} \sum_{x_{\mathbf{j}} \in \Omega^{d}}\left|\partial_{t} \phi_{\mathbf{j}}(t)\right|^{2}
$$

For all finite $T>0$ and mesh size $h>0$, it is easy to see that (6) holds for $C_{h}(T)$ sufficiently large. 
For the finite difference and $P_{1}$-classical finite element semi-discretizations of the wave equation on particular bounded domains like $d$-dimensional cubes, it is well-known from [10] that the corresponding observability constant blows-up as $h \rightarrow 0$, because of the pathological behavior of the spurious high frequency numerical solutions. In the context of the finite difference semi-discretization of the $1-d$ wave equation on a bounded interval, the observability constant is known to blow-up exponentially as $h \rightarrow 0$ for all $T>0$ (cf. [7]). As shown in [4], similar pathological high frequency phenomena have been observed in the context of the Strichartz dispersive estimates for the finite difference approximations of the Schrödinger equation.

In view of the formulation of the observability problem under consideration, our goal here is twofold. Firstly, to show the existence of numerical waves that are concentrated in space-time and then to give its precise asymptotic form in order to illustrate the added effect of the numerical dispersion with respect to the classical continuous wave equation. These solutions propagate according to the group velocity (cf. [9]), a notion that our construction contributes to make it precise. The group velocity of the high frequency numerical waves may be arbitrarily small, as the analysis of the dispersion diagram below shows.

Set $\Pi_{h}^{d}:=[-\pi / h, \pi / h]^{d}$. By applying the semi-discrete Fourier transform (SDFT) (see [9]) on (5) and denoting by $\widehat{\phi}^{h}(\xi, t)$ the SDFT of the solution $\vec{\phi}^{h}(t)$ of $(5)$, one obtains the following second-order ODE depending on the parameter $\xi$ :

$$
\begin{cases}\partial_{t}^{2} \widehat{\phi}^{h}(\xi, t)+\omega_{d, h}^{2}(\xi) \widehat{\phi}^{h}(\xi, t)=0, & \xi \in \Pi_{h}^{d}, t \in(0, T] \\ \widehat{\phi}^{h}(\xi, 0)=\widehat{\phi}^{h, 0}(\xi), & \partial_{t} \widehat{\phi}^{h}(\xi, 0)=\widehat{\phi}^{h, 1}(\xi), \xi \in \Pi_{h}^{d}\end{cases}
$$

where $\omega_{d, h}(\xi)$ is the multi-dimensional dispersion relation associated to $(5), \omega_{d, h}^{2}(\xi)=\frac{4}{h^{2}} \sum_{k=1}^{d} \sin ^{2}\left(\frac{\xi_{k} h}{2}\right)$, $\xi=\left(\xi_{k}\right)_{1 \leq k \leq d} \in \Pi_{h}^{d}$.

The semi-discrete rays of Geometric Optics corresponding to the semi-discrete problem (5) are straight lines of the form $x_{h}^{ \pm}(t)=x \pm \nabla \omega_{d, h}(\xi) t, x \in \mathbb{R}^{d}, \xi \in \Pi_{h}^{d}$, propagating with a group velocity, defined as the gradient $\nabla \omega_{d, h}(\xi)$. Note that $\left|\nabla \omega_{d, h}(\xi)\right|$ vanishes for all $\xi \in\{ \pm \pi / h, 0\}^{d} \backslash\{0\}$. This is in contrast with the behavior in the continuous case $(1)$, where the dispersion relation is $\omega_{d}(\xi)=|\xi|$ and the velocity of propagation along all rays is $\left|\nabla \omega_{d}(\xi)\right|=1$, for all $\xi \in \mathbb{R}^{d}$.

\section{Statement of the main result}

Let $T>0$ be given. Choose an arbitrary $x^{*} \in B^{d}(0,1)$ and consider a wave number $\eta_{0}=h \xi_{0} \in \Pi_{1}^{d} \backslash\{0\}$ such that the corresponding semi-discrete ray starting at $x^{*}$ and traveling with velocity $\left|\nabla \omega_{d, h}\left(\xi_{0}\right)\right|=$ $\left|\nabla \omega_{d, 1}\left(\eta_{0}\right)\right|$ does not enter the observation region in time $T$, i.e. $\left|x^{*}-t \nabla \omega_{d, 1}\left(\eta_{0}\right)\right|<1$, for all $t \in[0, T]$.

Consider $\gamma:=\gamma(h)>0$ such that

$$
\gamma>>1 \text { and } h \gamma<<1
$$

For $\phi \in \mathcal{S}\left(\mathbb{R}^{d}\right)$, the class of Schwartz functions in $\mathbb{R}^{d}, \widehat{\phi}$ being its continuous Fourier transform, consider the semi-discrete wave equation (5) with initial data $\left(\vec{\phi}^{h, 0}, \vec{\phi}^{h, 1}\right)$ whose SDFT is given by

$$
\widehat{\phi}^{h, 0}(\xi)=\frac{1}{i \omega_{d, h}(\xi)} \sqrt{\frac{2 \pi}{\gamma}} \widehat{\phi} \widehat{\phi}\left(\frac{\xi-\xi_{0}}{\gamma}\right) \exp \left(-i x^{*} \cdot\left(\xi-\xi_{0}\right)\right) \chi_{\Pi_{h}^{d}}(\xi) \text { and } \widehat{\phi}^{h, 1}(\xi)=i \omega_{d, h}(\xi) \widehat{\phi}^{h, 0}(\xi) \text {. }
$$

These data, in the physical space, correspond, roughly, to highly oscillationg Gaussian profiles. As shown by S. Ervedoza in [2], by a stationary phase like argument, one can show that for all $N \in \mathbb{N}$, there exists a constant $C_{N}(\widehat{\phi}, T)$ such that the observability constant $C_{h}(T)$ in $(6)$ satisfies $C_{h}(T) \geqslant C_{N}(\widehat{\phi}, T) \gamma^{2 N}$.

The initial data (9) are concentrated high frequency wave packets at the wave number $\xi=\xi_{0}=\eta_{0} / h$ with a width of order $1 / \gamma$, with $\gamma<<1 / h \rightarrow \infty$ as $h \rightarrow 0$. This limits drastically the spread of the wave 
packet and allows to concentrate its energy around the chosen ray and to reduce the added dispersive effects that the non-trivial Hessian matrix of the dispersion relation introduces. Simultaneously, by the uncertainty principle, this forces the wave packet, in the physical space, to have a spread factor of order $1 / \gamma>>h$, which is asymptotically larger than the characteristic size $h$ of the mesh. This is natural, in the sense that the numerical effects are only detected when an infinite number of nodes enter asymptotically in the determination of the data of the solution.

This example of wave packet clearly illustrates the classical effect due to the group velocity that is plotted in Figure 1 below. We plot in red the initial velocity $\phi^{1}(x)=\exp \left(-\gamma|x|^{2} / 2\right) \exp \left(i \xi_{0} x\right)$ (we also take $i|\xi| \widehat{\phi^{0}}=\widehat{\phi^{1}}$ ), with $h=0.005, \xi_{0} h=19 \pi / 20, \gamma=h^{-\alpha}, 2 \alpha=0.75$ in $d=1$ (left) and $d=2$ (right), which coincides, up to an exponentially small error (with respect to $h$ ), with the discrete initial data (9). In blue, we plot the time derivative of the solution of the continuous wave equation (1) and in black the one corresponding to the semi-discrete wave equation (5) with initial data (9) at time $t=1$. For $d=2$, we represent only the projection of these (continuous and discrete) solutions on the $x$-plane. This experiment shows that, as theory predicts, the semi-discrete wave packets propagate with a velocity that is much smaller than the one of the continuous wave equation.
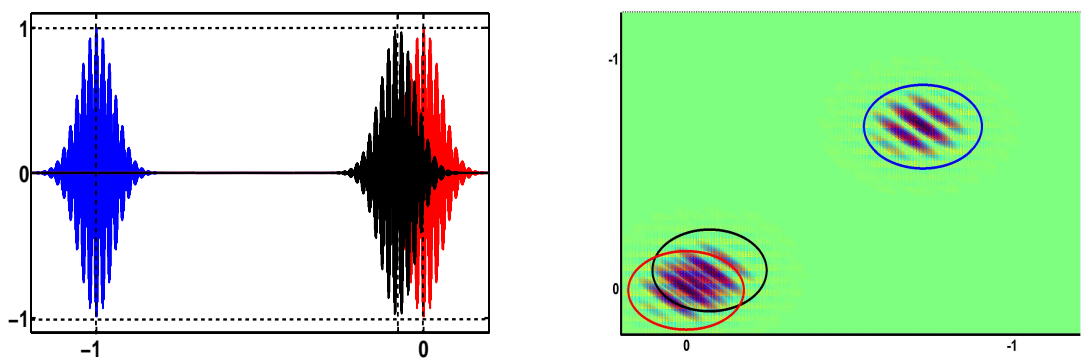

Figure 1. Transport of continuous versus discrete high frequency wave packets in dimension $d=1$ and $d=2$.

In the following, we analyze more precisely the behavior of these wave packets. To do this, we approximate the analytic dispersion relation $\omega_{d, h}(\xi)$ by a polynomial one.

Set $\Pi_{h}^{d}-\xi_{0}:=\left\{\xi-\xi_{0}, \xi \in \Pi_{h}^{d}\right\}$. The time derivative of the solution of (5) with initial data (9) is given by the following wave packet

$$
\partial_{t} \phi_{\mathbf{j}}(t)=\frac{1}{(2 \pi)^{d}} \int_{\mathbb{R}^{d}} \sqrt{\frac{2 \pi}{\gamma}}^{d} \widehat{\phi}(\xi / \gamma) \chi_{\Pi_{h}^{d}-\xi_{0}}(\xi) \exp \left(i t \omega_{d, h}\left(\xi+\xi_{0}\right)\right) \exp \left(i \xi \cdot\left(x_{\mathbf{j}}-x^{*}\right)\right) d \xi
$$

To simplify the presentation and, without loss of generality, we set $\xi_{0}=0$ and $x^{*}=0$. Since $\widehat{\phi} \in \mathcal{S}\left(\mathbb{R}^{d}\right)$, we may also neglect the characteristic function in (10). Setting $\omega(\xi)=\omega_{d, 1}\left(\xi+\eta_{0}\right)$ and $x=x_{\mathbf{j}}-x^{*}$, the wave packet (10) can be written as

$$
u(x, t)=\frac{1}{(2 \pi)^{d}} \int_{\mathbb{R}^{d}} \sqrt{\frac{2 \pi}{\gamma}}^{d} \widehat{\phi}\left(\frac{\xi}{\gamma}\right) \exp \left(\frac{i t}{h} \omega(\xi h)\right) \exp (i \xi \cdot x) d \xi .
$$

In view of the analyticity of $\omega(\eta)$, we can split it as $\omega(\eta)=L(\eta)+D(\eta)+R(\eta)$, where $L(\eta)=$ $\omega(0)+\nabla \omega(0) \cdot \eta$ is the linear part of $\omega, D$ is the second order term in the Taylor expansion of $\omega$ about $\eta=0$ and $R$ is the corresponding reminder, given explicitly by

$$
D(\eta)=\sum_{|\alpha|=2} \frac{1}{\alpha !} D^{\alpha} \omega(0) \eta^{\alpha}, R(\eta)=\sum_{|\alpha|=3} \frac{3}{\alpha !} \eta^{\alpha} \int_{0}^{1}(1-\lambda)^{2} D^{\alpha} \omega(\lambda \eta) d \lambda .
$$


Factoring it out the time dependent complex exponential generated by the zero order term in the Taylor expansion of the dispersion relation, we may decompose $u$ as $u \exp \left(-i t \omega_{d, 1}(0) / h\right)=v+v^{R}$, where

$$
v(x, t)=\frac{1}{(2 \pi)^{d}} \int_{\mathbb{R}^{d}} \sqrt{\frac{2 \pi}{\gamma}}^{d} \widehat{\phi}\left(\frac{\xi}{\gamma}\right) \exp \left(\frac{i t}{h} D(\xi h)\right) \exp (i \xi \cdot(x+t \nabla \omega(0))) d \xi
$$

and

$$
v^{R}(x, t)=\frac{1}{(2 \pi)^{d}} \int_{\mathbb{R}^{d}} \sqrt{\frac{2 \pi}{\gamma}}^{d} \widehat{\phi}\left(\frac{\xi}{\gamma}\right) \exp \left(\frac{i t}{h} D(\xi h)\right)\left(\exp \left(\frac{i t}{h} R(\xi h)\right)-1\right) \exp (i \xi \cdot(x+t \nabla \omega(0))) d \xi .
$$

Our main result determines the conditions on $\gamma$ and $\widehat{\phi}$, such that $v^{R}$ is a reminder term.

Theorem 2.1 For all $t>0$ and $\widehat{\phi} \in \mathcal{S}\left(\mathbb{R}^{d}\right)$, the following estimate holds:

$$
\frac{\left\|v^{R}(\cdot, t)\right\|_{L^{2}\left(\mathbb{R}^{d}\right)}^{2}}{\|u(\cdot, t)\|_{L^{2}\left(\mathbb{R}^{d}\right)}^{2}} \leq C(\widehat{\phi}) h^{4} \gamma^{6} t^{2}
$$

where $C(\widehat{\phi})=C\left\||\cdot|^{3} \widehat{\phi}\right\|_{L^{2}\left(\mathbb{R}^{d}\right)}^{2} /\|\widehat{\phi}\|_{L^{2}\left(\mathbb{R}^{d}\right)}^{2}$ and $C=\left(\sum_{|\alpha|=3} \frac{1}{\alpha !}\left\|D^{\alpha} \omega\right\|_{L^{\infty}(B(0, h \gamma))}\right)^{2}$

For a finite time interval $t \in[0, T]$, one can guarantee that $v^{R}$ is small in the sense of (12) when

$$
\gamma h^{2 / 3}<<1
$$

which is a more restrictive condition on $\gamma$ than (8), guaranteing that the energy concentrated outside the ray is polynomially small. Indeed, (12) yields an asymptotic description of the solution globally in space-time, i.e. $u \sim \exp (i t \omega(0) / h) v$. Intuitively, the scale (13) is motivated by the fact that $|R(\xi h) / h| \leq$ $\sqrt{C} h^{2}|\xi|^{3}$. For this to be asymptotically small, the width of the Fourier transform of the profile has to be limited by (13).

The function $v$ is a solution of the following PDE:

$$
\partial_{t} v=\nabla \omega(0) \cdot \nabla_{x} v-i h \sum_{|\alpha|=2} D^{\alpha} \omega(0) D_{x}^{\alpha} v
$$

This is a transport equation perturbed by an asymptotically small (as $h$ tends to zero) Schrödinger like second order term. The following result emphasizes that there exists solutions of (14) for which the relevant scale is

$$
\gamma h^{1 / 2}=1
$$

Theorem 2.2 There exist solutions of (14) admitting the following asymptotic expansion:

$$
v(x, t)=\sum_{j=0}^{\infty} h^{j / 2} a_{j}\left(\frac{x}{h^{1 / 2}}, \frac{t}{h^{1 / 2}}\right) \exp \left(i \xi \cdot \frac{\eta_{0}}{h^{1 / 2}}+i t \omega_{2}\left(\frac{\eta_{0}}{h^{1 / 2}}\right)\right),
$$

where $\eta_{0} \in \mathbb{R}^{d}$ is a fixed wave number, $\omega_{2}(\eta)=\nabla \omega(0) \cdot \eta+h \sum_{|\alpha|=2} \frac{1}{\alpha !} D^{\alpha} \omega(0) \eta^{\alpha}$ is the dispersion relation corresponding to (14) and $\left(a_{j}(x, t)\right)_{j \in \mathbb{N}}$ solve the following system of PDEs:

$$
\partial_{t} a_{0}=\nabla \omega(0) \cdot \nabla_{x} a_{0} \text { and } \partial_{t} a_{j+1}=\nabla \omega(0) \cdot \nabla_{x} a_{j+1}-i \sum_{|\alpha|=2} \frac{1}{\alpha !} D^{\alpha} \omega(0) \sum_{0 \leq \beta<\alpha}\left(i \eta_{0}\right)^{\beta} D_{x}^{\alpha-\beta} a_{j}, \forall j \in \mathbb{N} .
$$

By taking the support of the initial datum of $a_{0}$ to be compact, one can observe that $v$ is concentrated along a neighborhood of the ray of width $\sqrt{h}$. This scale $\sqrt{h}$ is critical due to the added dispersion that the Schrödinger like term introduces. Once the asymptotic expansion of $v$ is given as in (15), one 
immediately gets that of $u$ and therefore of $\partial_{t} \vec{\phi}$ and of $\vec{\phi}$. In this way, we get the asymptotic form of the high frequency wave packet.

This kind of expansion can be further developed, incorporating higher order terms of the Taylor expansion of the dispersion relation and a multiple-scale ansatz. This issue will be developed in detail in [3].

\section{Acknowledgements}

The authors acknowledge Sylvain Ervedoza for fruitful discussions. Both authors were partially supported by the Grant MTM2008-03541 of the MICINN, Spain, and the ERC Advanced Grant FP7-246775 NUMERIWAVES.

\section{References}

[1] C. Bardos, G. Lebeau, J. Rauch, Sharp sufficient conditions for the Observation, Control and Stabilization of waves from the boundary, SIAM J. Control and Optimization, 30 (1992), 1024-1065.

[2] S. Ervedoza, private communication.

[3] S. Ervedoza, A. Marica, E. Zuazua, Multiple-scale high frequecy concentrated wave packets for numerical approximation schemes of the wave equation, in preparation.

[4] L. Ignat, E. Zuazua, Numerical dispersive schemes for the nonlinear Schrödinger equation, SIAM. J. Numer. Anal., 47(2) (2009), 1366-1390.

[5] J.-L. Lions, Contrôlabilité exacte, perturbations et stabilization de systèmes distribués. Tome 1: Contrôlabilité exacte, Masson, 1988.

[6] A. Marica, Propagation properties for the discontinuous Galerkin and higher order finite element approximations of the wave equation, $\mathrm{PhD}$ Thesis, Universidad Autónoma de Madrid, in preparation.

[7] S. Micu, Uniform boundary controllability of a semi-discrete 1-D wave equation, Numer. Math., 91(4)(2002), $723-768$.

[8] J. Ralston, Gaussian beams and the propagation of singularities, in Studies in Partial Differential Equations, ed. by W. Littman, MAA Studies in Mathematics, 23(1983), 206-248.

[9] L.N. Trefethen, Group velocity in finite difference schemes, SIAM Review, 24(2)(1982), 113-136.

[10] E. Zuazua, Propagation, Observation, Control and Numerical Approximations of Waves, SIAM Review, 47(2)(2005), $197-243$.

[11] E. Zuazua, Exponential decay for the semilinear wave equation with localized damping in unbounded domains, J. Math. Pures Appl., 70 (1991), 513-529. 